\title{
Invited Speakers
}





\title{
Topology-Preserving Deletion of 1's from 2-, 3- and 4-Dimensional Binary Images
}

\author{
T. Yung Kong \\ Department of Computer Science \\ Queens College, CUNY \\ Flushing, NY 11367, U.S.A.
}

\begin{abstract}
In digital topology, a 1 in a binary image is said to be simple if its deletion from the image "preserves topology". Two (closely related) sets of necessary and sufficient conditions for a 1 in a 2-, 3- or 4dimensional binary image to be simple are established. The 4-dimensional cases of these results may be regarded as the principal contribution of this paper. A different discrete characterization of simple 1's in 2-, 3and 4-dimensional binary images, discovered by $\mathrm{A}$. W. Roscoe and the author, is also presented (without proof).
\end{abstract}

\section{$1 \quad n$-Xels and $n$-Images. Simple $n$-Xels.}

Let $n$ be any positive integer. An $n$-xel $q$ in Euclidean $n$-space, $\mathbf{R}^{n}$, is a closed unit $n$-dimensional (hyper)cube $q \subset \mathbf{R}^{n}$ whose $2^{n}$ vertices have integer coordinates. (More precisely, an $n$-xel in $\mathbf{R}^{n}$ is a Cartesian product of the form $\left[i_{1}, i_{1}+1\right] \times$ $\left[i_{2}, i_{2}+1\right] \times \ldots \times\left[i_{n}, i_{n}+1\right]$, where each of the $i$ 's is an integer.) In this paper, a pixel is a 2-xel in $\mathbf{R}^{2}$ and a voxel is a 3 -xel in $\mathbf{R}^{3}$. We define an $n$-dimensional binary image, or $n$-image, to be a finite set of $n$-xels in $\mathbf{R}^{n}$.

An $n$-image $I$ can of course be represented by a finite $n$-dimensional array of 1's and 0's in which each 1 represents an $n$-xel in $I$ and each 0 represents an $n$-xel that is not in $I$. For this reason each $n$-xel in an $n$-image $I$ may be called a 1 of $I$, and each $n$-xel that is not in $I$ may be called a 0 of $I$.

In this paper, we say that an $n$-xel in an $n$-image is simple if its removal from that image "preserves topology" in the sense of the following definition:

Definition 1. Let $q$ be an $n$-xel in an $n$-image I. $q$ is said to be simple in $I$ if the polyhedron $\bigcup I$ can be continuously deformed onto the polyhedron $U(I-\{q\})$ in such a way that throughout the deformation process:

1. All points that were originally in $q$ stay in $q$.

2. The points in $\bigcup(I-\{q\})$ do not move.

Equivalently, an element $q$ of an $n$-image $I$ is simple in $I$ if and only if the polyhedron $q$ can be continuously deformed over itself onto $q \cap \bigcup(I-\{q\})$ in such a way that all points in the latter set remain fixed throughout the deformation process. In the language of topology [1], $q$ is simple in $I$ if and only if there is a deformation retraction of $q$ onto $q \cap \bigcup(I-\{q\})$. 
This concept is illustrated in Figure 1. When $n=2$, it is equivalent to the standard concept of an "8-simple" (or "8-deletable") pixel. When $n=3$, it is equivalent to the standard concept of a "26-simple" voxel.
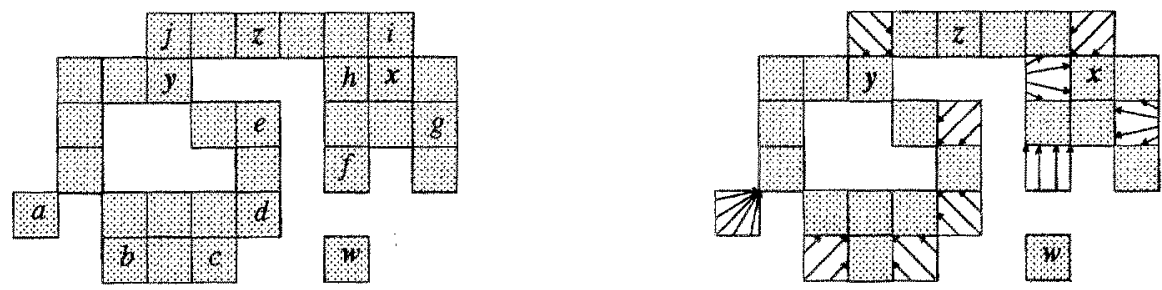

Fig. 1. The left-hand diagram shows ten simple pixels $\{a, b, \ldots, j\}$ and four nonsimple pixels $\{w, x, y, z\}$ in a 2-image. The right-hand diagram shows a continuous deformation of $\bigcup I$ onto $\bigcup(I-\{q\})$ in accordance with Definition 1 for each $q \in\{a, b, c, \ldots, j\}$.

Problem 2. How can one determine whether a given $n$-xel in an $n$-image $I$ is simple in $I$ ?

The problem is trivial in the case $n=1$. (A 1-xel $q$ in a 1-image $I$ is simple in $I$ if and only if exactly one of the two 1-xels that share an endpoint with $q$ is in I.) There are well known solutions in the cases $n=2$ and $n=3$-see section 2 . The main goal of this paper is to present a solution in the case $n=4$.

\section{Some Earlier Characterizations of Simple Xels in 2- and 3-Images.}

To place our work in context, we first recall solutions to the 2-d and 3 -d cases of the above problem.

The following well known characterization of simple pixels solves the 2 -d case of the problem:

Theorem 3 (cf. Rosenfeld [7]). A pixel $q$ in a 2-image $I$ is simple in $I$ if and only if both of the following conditions hold:

1. The union of the pixels in $I-\{q\}$ that share at least a vertex with $q$ (i.e., that "are 8-adjacent to $q$ ") is nonempty and connected.

2. q shares an edge with (i.e., "is 4-adjacent to") a poxel that is not in $I$.

It is readily confirmed that the simple pixels $a, b, \ldots, j$ in Figure 1 do indeed satisfy these conditions. The nonsimple pixel $x$ in Figure 1 fails condition 2, while the nonsimple pixels $w, y$ and $z$ fail condition 1 .

In the 3-d case, two different solutions to the problem are given by the next two theorems. The first of these theorems uses the concept of the Euler characteristic, which we define in section 3 . 
Theorem 4 (cf. Tsao and Fu [9]). A voxel $q$ in a 3-image $I$ is simple in I if and only if both of the following conditions hold:

1. The union of the voxels in $I-\{q\}$ that share at least a vertex with $q$ (i.e,, that "are 26-adjacent to $q$ ") is connected.

2. The union of the voxels in $I-\{q\}$ that share at least a vertex with $q$ has an Euler characteristic of 1 .

Theorem 5 (cf. Saha et al. [8], Malandain and Bertrand [6]). A voxel $q$ in a 3-image $I$ is simple in I if and only if the following conditions all hold:

1. The union of the voxels in $I-\{q\}$ that share at least a vertex with $q$ is nonempty and connected.

2. q shares a 2-d face with (i.e., "is 6-adjacent to") a voxel that is not in $I$.

3. For every two voxels $v$ and $v^{\prime}$ that share a 2-d face with $q$ and are not in $I$, there is a sequence $v=v_{0}, v_{1}, \ldots, v_{k}=v^{\prime}$ of voxels that are not in $I$ in which each $v_{i}(1 \leq i \leq k)$ shares at least an edge with $q$ (i.e., "is 18-adjacent to $\left.q^{\prime \prime}\right)$ and shares a 2-d face with $v_{i-1}$.

The three theorems above can in fact be deduced from Corollary 8 below without too much work.

Our main theorem (Theorem 7 below) will solve the problem in the case $n=4$. Another version of the same result (Theorem 9 ) will give a practical algorithm for determining whether a 4-xel in a 4-image $I$ is simple in $I$.

\section{Xels and Their Faces. Xel Complexes. Boundary $(q)$.}

In section 1 we defined the concept of an $n$-xel in $\mathbf{R}^{n}$ for $n \geq 1$. More generally, for any integer $k \geq 0$ we define a $k$-dimensional $x e l$, or $k$-xel, to be a closed unit $k$-cube in a Euclidean space $\mathbf{R}^{n}$ for some $n \geq \max (k, 1)$. We use the term xel to mean a $k$-xel for some $k \geq 0$. Thus a xel is a Cartesian product $J_{1} \times J_{2} \times \ldots \times J_{n}$ where $n \geq 1$ and each $J$ either is a closed unit interval with integer endpoints or is a set consisting of a single integer; the xel is a $k$-xel if exactly $k$ of the $J$ 's are unit intervals. Note that a 0 -xel is a singleton set $\{p\}$, where $p$ is a point with integer coordinates.

If $X$ and $Y$ are xels such that $X \subseteq Y$, then we say the xel $X$ is a face of the xel $Y$. A xel complex is a finite set $K$ of xels such that every face of each xel in $K$ is itself an element of $K$. A subcomplex of a xel complex $K$ is a set $L \subseteq K$ such that $L$ is itself a xel complex; a proper subcomplex of $K$ is a subcomplex of $K$ other than $K$ itself. The boundary of a xel $q$, denoted by Boundary $(q)$, is the xel complex consisting of all the faces of $q$ except $q$ itself. For example, if $q$ is a voxel then Boundary $(q)$ consists of eight 0-xels, twelve 1-xels and six 2-xels.

The Euler characteristic of a xel complex $K$ is defined to be the following integer: $\sum_{k=0}^{\infty}(-1)^{k}$ (the number of $k$-xels in $K$ ). There are only finitely many nonzero terms in this sum since xel complexes are finite sets of xels. A well known property of the Euler characteristic is that if $K_{1}$ and $K_{2}$ are xel complexes such 
that the polyhedra $\bigcup K_{1}$ and $\bigcup K_{2}$ are homeomorphic (i.e., topologically equivalent), then $K_{1}$ and $K_{2}$ have the same Euler characteristic. If $S$ is any set that is homeomorphic to $\bigcup K$ for some xel complex $K$, then the Euler characteristic of $S$ is defined to be the Euler characteristic of $K$.

Proposition 6. Let $q$ be an n-xel. Then the Euler characteristic of Boundary $(q)$ is 2 if $n$ is odd and is 0 if $n$ is even.

Proof. For all integers $k \geq 0$, let $c_{k}$ be the number of $k$-xels in Boundary $(q)$. Now $q=\left[i_{1}, i_{1}+1\right] \times\left[i_{2}, i_{2}+1\right] \times \ldots \times\left[i_{n}, i_{n}+1\right]$ for some integers $i_{1}, i_{2}, \ldots, i_{n}$, and each $k$-xel in Boundary $(q)$ is obtained by replacing just $n-k$ of the intervals $\left[i_{j}, i_{j}+1\right]$ with $\left\{i_{j}\right\}$ or $\left\{i_{j}+1\right\}$. Hence $c_{k}=2^{n-k} C(n, n-k)=2^{n-k} C(n, k)$ for $0 \leq k<n$. Evidently, $c_{k}=0$ for $k \geq n$. Thus $c_{k}$ is the coefficient of $t^{k}$ in the binomial expansion of $p_{n}(t)=(2+t)^{n}-t^{n}$. So the Euler characteristic of Boundary $(q)$, which is $\sum_{k=0}^{\infty}(-1)^{k} c_{k}$, is equal to $p_{n}(-1)=1-(-1)^{n}$.

\section{Schlegel Diagrams.}

Let $n$ be a positive integer, $q$ an arbitrary $n$-xel, and $K$ an arbitrary proper subcomplex of Boundary $(q)$.

A Schlegel diagram of $K$ represents $K$ in $\mathbf{R}^{n-1}$. This is particularly useful in the case where $q$ is a 4-xel, because it allows us to think in three dimensions rather than four.

To construct a Schlegel diagram of $K$, let $f$ be an $(n-1)$-dimensional face of $q$ that is not in $K$, let a be some point not in $q$ whose orthogonal projection on $q$ is the centroid of the face $f_{2}$ and let $P$ be an $(n-1)$-dimensional hyperplane parallel to $f$ such that $q$ lies between $P$ and the $(n-1)$-dimensional hyperplane that is the affine hull of $f$. Consider a projection of $U($ Boundary $(q)-\{f\})$, with $a$ as the "light source", onto $P$. Let $\pi_{a, P}: \bigcup(B o u n d a r y(q)-\{f\}) \rightarrow \mathbf{R}^{n-1}$ be the map induced by this projection. More precisely, for each $x \in U(\operatorname{Boundary}(q)-\{f\})$ let $g(x)$ be the point at which the line through $a$ and $x$ meets the hyperplane $P$, and let $\pi_{a, P}(x)$ be the point in $\mathbf{R}^{n-1}$ obtained from the point $g(x)$ by omitting the coordinate whose axis is orthogonal to $f$ and $P$. Then the set $\left\{\pi_{a, P}[z] \mid z \in K\right\}$ will be called a Schlegel diagram of $K$.

The map $\pi_{a, P}$ is a homeomorphism of $U(B o u n d a r y(q)-\{f\})$ onto its image. Moreover, it is readily confirmed that this map can be extended to a homeomorphism of $\cup B o u n d a r y(q)$ onto $\mathbb{R}^{n-1} \cup\{\infty\}$. Here $\infty$ denotes a single point at infinity. It follows that if $S_{K}$ is a Schlegel diagram of $K$ then:

(1) $\bigcup S_{K}$ is homeomorphic to $\bigcup K$.

(2) $\left(\mathbf{R}^{n-1} \cup\{\infty\}\right)-\bigcup S_{K}$ is homeomorphic to $\bigcup$ Boundary $(q)-\bigcup K$.

(2) implies that $\mathbf{R}^{n-1}-\bigcup S_{K}$ is comnected if and only if $U$ Boundary $(q)-\cup K$ is connected.

Figure 2 shows a Schlegel diagram of Boundary $(q)-\{f\}$ in the case where $q$ is a voxel and $f$ is one of the six 2 -d faces of $q$. In the case where $q$ is a 4-xel and $f$ is a 3 -d face of $q$, it is not hard to verify that the structure of a Schlegel diagram of Boundary $(q)-\{f\}$ is as shown in Figure 3 . 

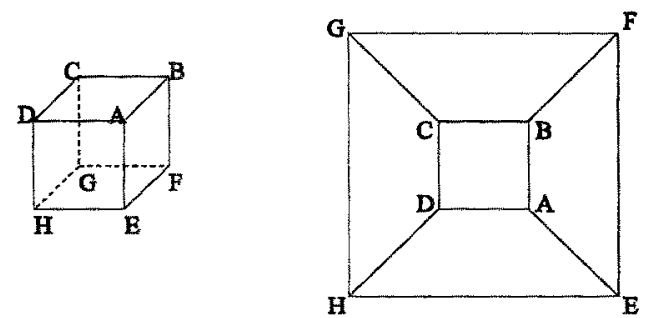

Fig. 2. A voxel $q$ and a Schlegel diagram of Boundary $(q)-\{f\}$, where $f$ is the face of $q$ whose vertices are $E, F, G$ and $H$.

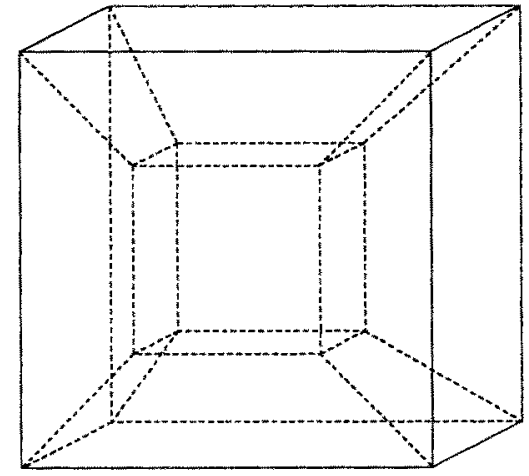

Fig. 3. A Schlegel diagram of Boundary $(q)-\{f\}$ in the case where $q$ is a 4 -xel and $f$ is a 3-d face of $q$.

\section{$5 \operatorname{Attach}(q, I):$ The Attachment of an $n$-Xel $q$ in an $n$-Image $I$. The Main Theorem.}

Let $q$ be an $n$-xel in an $n$-image $I$. The attachment of $q$ in $I$ is the (possibly empty) xel complex Boundary $(q) \cap \bigcup\{$ Boundary $(x) \mid x \in I-\{q\}\}$. It is denoted by $\operatorname{Attach}(q, I)$. Figure 4 illustrates this concept.

We may think of $I$ as being obtained by "gluing" $q$ onto $\bigcup(I-\{q\})$; in that case UAttach $(q, I)$ is the set of points of UBoundary $(q)$ at which glue may usefully be applied!

Note that $\bigcup \operatorname{Attach}(q, I)=q \cap \bigcup(I-\{q\})$. Thus $q$ is simple in $I$ if and only if $q$ can be continuously deformed over itself onto $\bigcup \operatorname{Attach}(q, I)$ in such a way that the points in $\bigcup \operatorname{Attach}(q, I)$ remain fixed throughout the deformation process.

We are now ready to state the main result of this paper. For $1 \leq n \leq 4$, the result gives conditions on $\operatorname{Attach}(q, I)$ that are necessary and sufficient for an $n$-xel $q$ to be simple in an $n$-image $I$. 


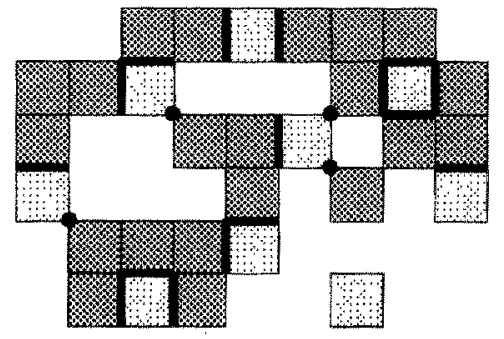

Attachments of Nine Pixels in a 2-Image.

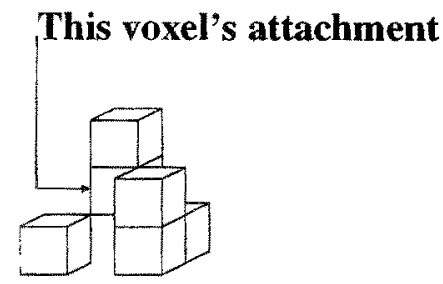

... looks like this:

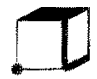

Here is a Schlegel diagram of the attachment:
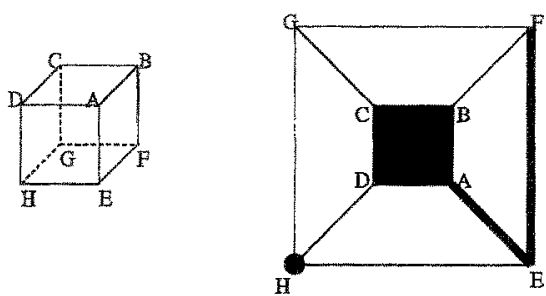

Fig. 4. Examples of attachments.

Theorem 7 (The Main Theorem). Let $q$ be an n-xel in an $n$-image I, where $1 \leq n \leq 4$. Then $q$ is simple in $I$ if and only if the following conditions all hold:

(a) UAttach $(q, I)$ is nonempty and connected.

(b) UBoundary $(q)-\bigcup \operatorname{Attach}(q, I)$ is nonempty and connected.

(c) UAttach $(q, I)$ is simply connected.

Condition (c) of the Main Theorem is needed only when $n=4$, because (a) and (b) imply (c) when $n \leq 3$. Thus for $n \leq 3$ we have the following simpler characterization of simple $n$-xels:

Corollary 8. Let $q$ be an $n$-xel in an $n$-image $I$, where $1 \leq n \leq 3$. Then $q$ is simple in I if and only if both of the following conditions hold:

(a) UAttach $(q, I)$ is nonempty and connected.

(b) UBoundary $(q)-\bigcup \operatorname{Attach}(q, I)$ is nonempty and connected.

This is the same as the characterization of simple 2- and 3-xels given in Theorem 2.10 of [3], though simpleness was defined in a slightly different (but, in fact, equivalent) way in that paper.

Figure 5 shows a violation of condition (b) of Corollary 8. Figure 6 shows a violation of condition (c) of Theorem 7. In both cases $q$ is nonsimple in $I$. The voxel whose attachment is shown in the right-hand diagram of Figure 4 violates condition (a) of Corollary 8 and is therefore nonsimple. 

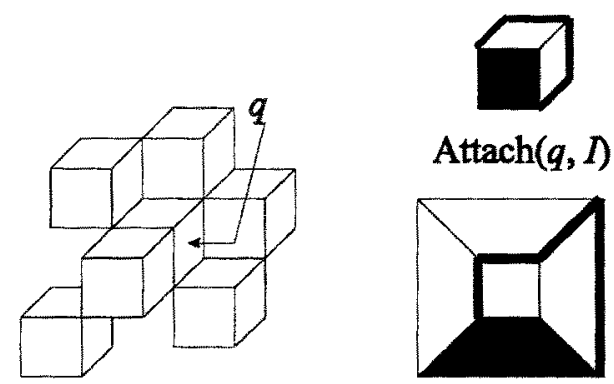

\section{UBoundary $(q)$ - UAttach $(q, D)$ is disconnected. Hence $q$ is nonsimple.}

Fig. 5. An example of how Attach $(q, I)$ can violate condition (b) of Corollary 8 in the case $n=3$.

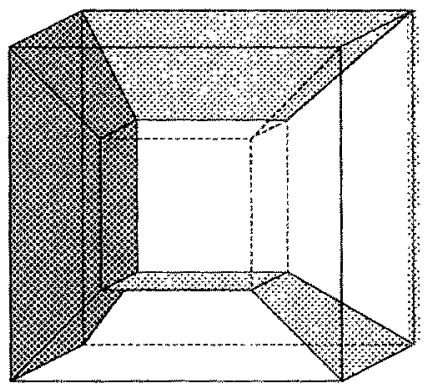

3-xel

. $k$-xel, $k \leq 2$

\section{UAttach $(q, I)$ is} not simply connected. Hence $q$ is nonsimple.

Fig. 6. A Schlegel diagram of an attachment that violates condition (c) of the Main Theorem in the case $n=4$.

In the following version of the Main Theorem, condition (c) has been changed to a condition on the Euler characteristic of $\operatorname{Attach}(q, I)$. As the Euler characteristic is easily computed, this version of the Main Theorem gives a very practical algorithm for determining whether a 1 in a 4-dimensional binary image is simple.

Theorem 9 (The Main Theorem, version 2). Let $q$ be an $n$-xel in an $n$ image $I$, where $1 \leq n \leq 4$. Then $q$ is simple in $I$ if and only if the following conditions all hold:

(a) UAttach $(q, I)$ is connected.

(b) UBoundary $(q)-\bigcup \operatorname{Attach}(q, I)$ is connected.

(c) The Euler characteristic of $\operatorname{Attach}(q, I)$ is 1 .

We claim that Theorems 7 and 9 are equivalent. In verifying this we may assume $\bigcup \operatorname{Attach}(q, I)$ and $\bigcup B$ oundary $(q)-\bigcup \operatorname{Attach}(q, I)$ are both connected; otherwise (a) or (b) fails in both theorems. 
If $\operatorname{Attach}(q, I)$ is empty then condition (a) of Theorem 7 and condition (c) of Theorem 9 fail. If $\operatorname{Attach}(q, I)=$ Boundary $(q)$ then condition (b) of Theorem 7 and condition (c) of Theorem 9 fail-the latter fails by Proposition 6 . Thus we may further assume that $\operatorname{Attach}(q, I)$ is nonempty and $\operatorname{Attach}(q, I) \neq$ Boundary $(q)$, so that conditions (a) and (b) of both theorems hold.

To justify the claim, we must now show that the conditions (c) of the two theorems are equivalent - that the Euler characteristic of $\operatorname{Attach}(q, I)$ is 1 if and only if the polyhedron $\bigcup A t \operatorname{tach}(q, I)$ is simply connected.

It is a well known property of bounded polyhedra in 3-space that for any such polyhedron $A$

$$
\chi(A)=h_{0}(A)-h_{1}(A)+h_{2}(A)
$$

where $\chi(A)$ denotes the Euler characteristic of $A, h_{0}(A)$ denotes the number of connected components of $A, h_{2}(A)$ denotes the number of 3-d cavities in $A$, and where $h_{1}(A)$ (which essentially denotes the number of annulus/doughnut-type holes in $A$ ) is 0 if and only if $A$ is simply connected [4].

Let $A$ be the union of a Schlegel diagram of $\operatorname{Attach}(q, I)$. As $\bigcup \operatorname{Attach}(q, I)$ is connected, so is $A$. As $U$ Boundary $(q)-\bigcup \operatorname{Attach}(q, I)$ is connected, so is $\mathbf{R}^{3}-A$; thus the polyhedron $A$ has no 3-d cavities. So $h_{0}(A)=1$ and $h_{2}(A)=0$ in equation (1), which implies that $\chi(A)=1-h_{1}(A)$. Hence $\chi(A)$ has value 1 if and only if $A$ is simply connected, and so the Euler characteristic of $\operatorname{Attach}(q, I)$ is 1 if and only if $\cup \operatorname{Attach}(q, I)$ is simply connected.

\section{Proof of the "Only If" Part of the Main Theorem.}

Let $q$ be a simple xel in an $n$-image $I$, where $n \leq 4$. In this section we show that $\operatorname{Attach}(q, I)$ must satisfy conditions $(\mathrm{a}-\mathrm{c})$ of Theorem 7 .

Continuous deformation preserves many topological properties, including connectedness, simple connectedness, and the Euler characteristic. As the nxel $q$ is an $n$-cube, it is convex and can therefore be continuously deformed to a point. So, just like a point, $q$ has the following properties:

(q1) $q$ is nonempty and connected.

(q2) $q$ is simply connected.

(q3) The Euler characteristic of $q$ is 1 .

As the $n$-xel $q$ is simple in $I, q$ can be continuously deformed over itself onto $\bigcup \operatorname{Attach}(q, I)$. Hence $(\mathrm{q} 1-\mathrm{q} 3)$ imply

(A1) $\bigcup \operatorname{Attach}(q, I)$ is nonempty and connected.

(A2) U Attach $(q, I)$ is simply connected.

(A3) The Euler characteristic of $U \operatorname{Attach}(q, I)$ is 1 .

Note that A3 implies UBoundary $(q)-U \operatorname{Attach}(q, I)$ is nonempty, by Proposition 6.

To prove the "only if" part of the Main Theorem, it remains only to show that $\bigcup$ Boundary $(q)-\bigcup \operatorname{Attach}(q, I)$ is connected. We will give an elementary proof of this, based on equation (1) of the previous section. 
The result is evidently true if $n=1$. If $n=2$, then it follows from A1. If $n=3$ then it follows from A2. In the case $n=4$, let $A$ be the union of a Schlegel diagram of $\operatorname{Attach}(q, I)$. Then $A$ is connected (by A1), simply connected (by A2), and has an Euler characteristic of 1 (by A3). Thus in equation (1) above we have $h_{0}(A)=1, h_{1}(A)=0$, and $\chi(A)=1$, so that $1=1-0+h_{2}(A)$, which implies $h_{2}(A)=0$. Hence $\mathbf{R}^{3}-A$ is connected, and so $\bigcup$ Boundary $(q)-\bigcup \operatorname{Attach}(q, I)$ is connected, as required.

This argument establishes the "only if" part of the Main Theorem.

\section{Proof of the "If" Part of the Main Theorem.}

We now prove that if the three conditions of Theorem 7 hold, then $q$ can be continuously deformed onto $\bigcup \operatorname{Attach}(q, I)$. We prove this using a classical discrete deformation process for xel complexes called collapsing. As we will see in section 7.2, this process has the property that if a xel complex $K \subseteq$ Boundary $(q)$ can be collapsed to a "trivial" complex consisting of a single 0-xel, then the xel $q$ can be continuously deformed to $\bigcup K$. We prove that if the three conditions of Theorem 7 all hold then $\operatorname{Attach}(q, I)$ can indeed be collapsed to a trivial complex, so that $q$ can be continuously deformed onto $\bigcup \operatorname{Attach}(q, I)$, as required.

\subsection{The * Operation. Free Faces of a Complex. Collapsing.}

Let $q$ be any xel and let $c$ be the center of $q$. For every xel $x$ in Boundary $(q)$, we define $c * x$ to be the convex hull of $\{c\} \cup x$; we say that $c * x$ is $(k+1)$-dimensional if $x$ is a $k$-dimensional xel. For every subcomplex $K$ of Boundary $(q)$, we define $c * K=\{c * x \mid x \in K\} \cup\{c\} \cup K$. For every xel $y$ in Boundary $(q)$, the faces of the polyhedron $c * y$ are:

1. The xel $y$.

2. The elements of $c *$ Boundary $(y)$.

3. $c * y$ itself.

If $w=c * y$ then the boundary of $w$, denoted by Boundary $(w)$, is the set of all faces of $w$ other than $w$ itself. A subset $Z$ of $c *$ Boundary $(q)$ is called a complex if it satisfies the condition that, for each $z \in Z$, every face of $z$ is also an element of $Z$.

In the rest of this paper we use the term complex to mean either a complex of the kind we have just defined, or a xel complex. (Both of these are special cases of the standard concept of a polyhedral complex, but they are the only cases we will use.)

We now define collapsing. This is a standard tool of geometric combinatorial topology.

Let $x$ be a maximal element of a complex $K$ (i.e., an element of $K$ that is not included in any higher-dimensional element of $K)$ and let $d$ be the dimension of $x$. If $d \geq 1$ then a free face of $x$ (in $K$ ) is a $(d-1$ )-dimensional face $f$ of $x$ that 
is not included in any element of $K-\{x, f\}$. A free face of $K$ is an element of $K$ that is a free face of $z$ in $K$ for some maximal element $z$ of $K$.

If $f$ is a free face of an element $x$ of a complex $K$, then the process of removing both $x$ and $f$ from $K$ is called an elementary collapse of $K$, at $f$, to the complex $K-\{x, f\}$. A complex $K$ is said to collapse to a complex $L \subseteq K$ if there is a (possibly empty) sequence of elementary collapses that transforms $K$ to $L$. A complex $K$ is said to be collapsible if $K$ collapses to a complex that consists only of a single 0-xel. These concepts are illustrated in Figure 7.

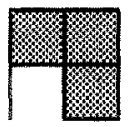

0

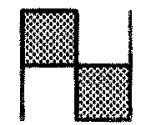

1

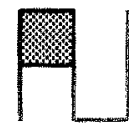

2

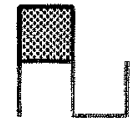

3

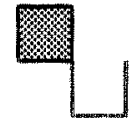

4

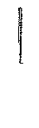

10

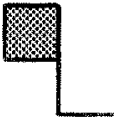

5

11

Fig. 7. Each of the complexes $1-11$ is obtainable from its predecessor by an elementary collapse. Since the final complex in this sequence consists of a single 0-xel, each of the complexes $0-11$ is collapsible.

Collapsing and collapsibility are of interest to us largely because of the following well known fact:

Proposition 10. If a complex $K$ collapses to a complex $L$, then there is a continuous deformation of $\bigcup K$ to $\bigcup L$ that never moves the points of $\bigcup L$.

Proof (sketch). It is enough to show that such a continuous deformation exists whenever $L$ is obtainable from $K$ by a single elementary collapse. Suppose $L$ is obtained from $K$ by an elementary collapse at a free face $f$ of an element $q \in K$. Let $d$ be the dimension of $q$ and let $f_{0}, f_{1}, \ldots, f_{k}$ be an enumeration of the $(d-1)$-dimensional faces of $q$, where $f_{0}$ is the free face $f$. For $0 \leq i \leq k$ let $P_{i}$ be the affine hull of $f_{i}$, and let $H_{i}$ be the closed $d$-dimensional half-space in the affine hull of $q$ such that $H_{i}$ is bounded by the hyperplane $P_{i}$ and $q \subseteq H_{i}$. It can be shown that the half-spaces $H_{i}$ exist, and that $q=\bigcap_{i=0}^{i=k} H_{i}$.

Let $b$ be some point in $\bigcap_{i=1}^{i=k}\left(H_{i}-P_{i}\right)-H_{0}$. For each point $x$ in $q$, it is readily confirmed that the straight line through $b$ and $x$ meets $U($ Boundary $(q)-\{f\})$ at just one point; let $g(x)$ be that point. For each point $x$ in $\cup K-q$, let $g(x)=x$. It is readily confirmed that this defines a continuous map $g: \bigcup K \rightarrow \bigcup L$. For each $x$ in $U K$ and each $t \in[0,1]$, let $H(x, t)=\operatorname{tg}(x)+(1-t) x$. It is evident 
that $H$ is continuous, that $H(x, 0)=x$ and $H(x, 1) \in \bigcup L$ for all $x$ in $\bigcup K$, and that if $x \in \bigcup L$ then $H(x, t)=x$ for all $t$ in $[0,1]$. Thus $H$ defines a "continuous deformation of $\bigcup K$ to $\bigcup L$ that never moves the points of $\bigcup L "$.

\subsection{A Sufficient Condition for Simpleness.}

We will deduce the "if" part of the Main Theorem from the following sufficient condition for a xel to be simple. Note that in the following proposition $n$ is not restricted to being $\leq 4$.

Proposition 11. Let $n$ be any positive integer and let $q$ be an $n$-xel in an $n$ image I such that $\operatorname{Attach}(q, I)$ is collapsible. Then $q$ is simple in $I$.

Our proof of this proposition depends on two lemmas.

Lemma 12. Let $c$ be the center of an n-xel $q$. Then for every xel complex $K \subseteq$ Boundary $(q), c *$ Boundary $(q)$ collapses to $c * K$.

Proof. If $x$ is a xel in Boundary $(q)$ that is not in $K$, then each xel in Boundary $(q)$ that $x$ is a face of is not in $K$. Starting with $c *$ Boundary $(q)$, we can perform elementary collapses at the $(n-1)$-xels in Boundary $(q)$ that are not in $K$, then at the $(n-2)$-xels in Boundary $(q)$ that are not in $K$, then at the $(n-3)$-xels in Boundary $(q)$ that are not in $K$, and so on until elementary collapses have been done at all the xels in Boundary $(q)$ that are not in $K$. We are then left with $c * K$, as required.

Lemma 13. Let $c$ be the center of an $n$-xel $q$, and let $K$ be a collapsible subcomplex of Boundary $(q)$. Then the complex $c * K$ collapses to $K$.

Proof. As $K$ is collapsible, there is a sequence of elementary collapses that transforms $K$ to a complex consisting of a single 0 -xel $p$. Let $m$ be the number of elementary collapses in this sequence, and let $K=K_{0}, K_{1}, \ldots, K_{m}=\{p\}$ be the sequence of complexes we go through. Let $f_{0}, f_{1}, \ldots, f_{m-1}$ be the free faces at which the elementary collapses occur (so that $f_{i}$ is a free face of $K_{i}$ ). Then we can collapse $c * K$ to $K$ using the sequence of $m+1$ elementary collapses at the free faces $c * f_{0}, c * f_{1}, \ldots, c * f_{m-1}, c$.

Proof of Proposition 11. On putting $K=\operatorname{Attach}(q, I)$ in Lemmas 12 and 13 we deduce that:

(A) $c *$ Boundary $(q)$ collapses to $c * \operatorname{Attach}(q, I)$.

(B) $c * \operatorname{Attach}(q, I)$ collapses to $\operatorname{Attach}(q, I)$ (since $\operatorname{Attach}(q, I)$ is collapsible).

It follows from (A) and (B) that $c *$ Boundary $(q)$ collapses to Attach $(q, I)$. Hence (by Proposition 10) there is a continuous deformation of $q=\bigcup(c * \operatorname{Boundary}(q))$ to $\bigcup \operatorname{Attach}(q, I)$, and $q$ is simple. 


\subsection{Completion of the Proof of the Main Theorem.}

Let $q$ be an $n$-xel in an $n$-image $I$, where $n \leq 4$, such that conditions $(\mathrm{a}-\mathrm{c})$ of the Main Theorem are satisfied. In view of Proposition 11, we can complete the proof of the Main Theorem by showing that $\operatorname{Attach}(q, I)$ must be collapsible,

The result is trivially valid when $n=1$. When $n=2$ the only possibilities for $\operatorname{Attach}(q, I)$ are those shown in Figure 8. In each case Attach $(q, I)$ is evidently collapsible. So the Main Theorem is true for $n=2$.

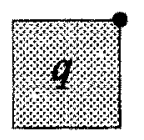

No edge

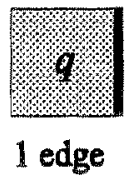

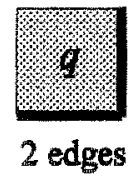

Fig. 8. Possible forms of Attach $(q, I)$ in the case where $q$ is a pixel that satisfies conditions $(a-c)$ of the Main Theorem.

Now suppose $n=3$ or 4 . Since $q$ satisfies conditions $(a-c)$ of the Main Theorem, the following conditions hold when $P=\operatorname{Attach}(q, I)$ :

(A) $U P$ is nonempty and connected.

(B) UBoundary $(q)-\cup P$ is nonempty and connected.

(C) $\bigcup P$ is simply connected.

We now show that any xel complex $P \subseteq B$ Boundary $(q)$ which satisfies $(A),(B)$ and (C) is collapsible. Indeed, suppose the complex $P$ is a minimal counterexample to this assertion. Then $P$ satisfies $(A-C)$ but is not collapsible. (B) implies that $P \neq \operatorname{Boundary}(q)$. Since elementary collapses preserve properties $(A-C)$, and $P$ is a minimal counterexample, $P$ cannot have any free face.

The Case $n=3$. In this case it is evident (e.g., from Figure 2) that $P$ cannot contain any 2 -xel, for otherwise one of the 2 -xels in $P$ would have a free face in $P$. If $P$ contains a 1-xel, then since each endpoint of a 1 -xel in $P$ must be an endpoint of another 1-xel in $P$ (otherwise it would be a free face, contradiction) the 1-xels in $P$ contain a cycle. But then $U P$ is not simply connected, which contradicts (C). Thus $P$ contains only 0 -xels. Since $U P$ is nonempty and connected (by (A)), $P$ consists of exactly one 0 -xel. But then $P$ is (trivially) collapsible after all, which is a contradiction. Thus the minimal counterexample $P$ cannot exist, and so the Main Theorem holds when $n=3$.

The Case $n=4$. In this case we see from Figure 3 that the minimal counterexample $P$ cannot contain any 3-xel, for otherwise one of the 3-xels in $P$ would have a free face in $P$. If $P$ also contains no 2 -xel, then we can use the same argument as we just used in the case $n=3$ to derive a contradiction. 
Now we suppose $P$ contains a $2-x e l$, and deduce another contradiction. Let Schlegel $(P)$ be a Schlegel diagram of $P$.

It is convenient to refer to 1 -xels as edges and 0 -xels as vertices. Each edge in Boundary $(q)$ is incident on just three 2-xels of Boundary $(q)$, and hence on zero, one, two or three 2-xels in $P$. No edge is incident on just one 2-xel in $P$ (otherwise the edge would be a free face of that $2-x e l$ ). We claim that no edge is incident on three 2-xels in $P$.

Assume for the moment that we can prove this. Then each edge is incident on no 2-xel in $P$ or just two 2-xels in $P$. It follows from a standard "Jordan surface" theorem - the Alexander Duality Theorem (see, e.g., [2]) - that the set $\mathbf{R}^{3}$-USchlegel $(P)$ is disconnected, so that $\bigcup \operatorname{Boundary}(q)-\bigcup P$ is disconnected, contrary to $(\mathrm{B})$.

It remains only to justify our claim that no edge is incident on three 2-xels in $P$. Call an edge a 3-edge of $P$ if it is incident on three 2-xels in $P$, and call the corresponding element of $\operatorname{Schlegel}(P)$ a 3-edge of $\operatorname{Schlegel}(P)$. (Note that all 2-xels of Boundary $(q)$ that are incident on a 3-edge of $P$ are in $P$.) We want to show that no 3 -edges of $P$ exist. We first prove:

Lemma 14. Let $y$ be any 3-xel in Boundary $(q)$. Then at most four of the six 2-xels that are faces of $y$ can be in P.

An immediate corollary of this lemma is:

Corollary 15. In each of $(a, b, c)$ below, the four thick black edges cannot all be 3-edges of Schlegel $(P)$ :

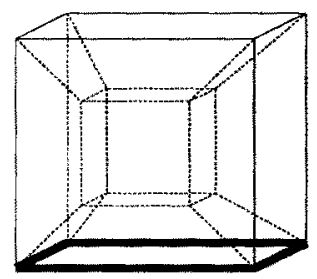

(a)

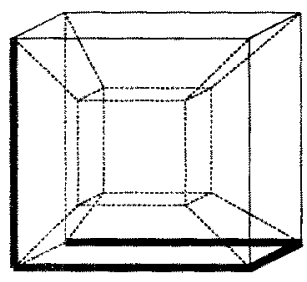

(b)

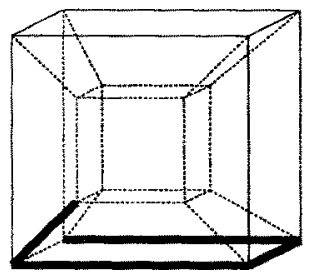

(c)

Proof of Lemma 14. It will probably be helpful to refer to Figure 3 in following this proof.

Since we know $P$ contains no 3 -xel, there must be a 2 -d face $f_{1}$ of $y$ that is not in $P$, for otherwise $U$ Boundary $(q)-\bigcup P$ would not be connected. Suppose the other five 2-d faces of $y$ are all in $P$. Let $y_{1}$ be the 3-xel in Boundary $(q)$ that shares the face $f_{1}$ with $y$, let $f_{2}$ be the 2-d face of $y_{1}$ opposite $f_{1}$, let $y_{2}$ be the 3-xel in Boundary $(q)$ that shares the face $f_{2}$ with $y_{1}$, let $f_{3}$ be the 2-d face of $y_{2}$ opposite $f_{2}$, let $y_{3}$ be the 3 -xel in Boundary $(q)$ that shares the face $f_{3}$ with $y_{2}$, and let $f_{4}$ be the $2-\mathrm{d}$ face of $y_{3}$ opposite $f_{3}$. (Thus $f_{4}$ is the $2-\mathrm{d}$ face of $y$ opposite $f_{1}$.)

Since the four 2-d faces of $y$ that are adjacent to $f_{1}$ are all in $P$ but none of them has a free face in $P$, and since $f_{1} \notin P$, the four $2-\mathrm{d}$ faces of $y_{1}$ that are 
adjacent to $f_{1}$ and $f_{2}$ are all in $P$. Hence $f_{2} \notin P$, for otherwise $\bigcup$ Boundary $(q)-$ UP would not be connected. So since none of the four 2-d faces of $y_{1}$ that are adjacent to $f_{2}$ has a free face in $P$, the four 2-d faces of $y_{2}$ that are adjacent to $f_{2}$ and $f_{3}$ are all in $P$. Hence $f_{3} \notin P$ (as $\cup$ Boundary $(q)-U P$ is connected) and the four 2-d faces of $y_{3}$ that are adjacent to $f_{3}$ and $f_{4}$ are all in $P$ (as $P$ has no free face). But this implies that UP separates the interiors of the 3-xels $y, y_{1}$, $y_{2}$ and $y_{3}$ from the interiors of the other four 3-xels of Boundary $(q)$, contrary to the hypothesis that $\bigcup$ Boundary $(q)-\bigcup P$ is connected. So at most four of the 2-d faces of $y$ can be in $P$.

Our next step in proving that no 3-edges of $P$ exist is:

Lemma 16. No vertex of Boundary $(q)$ is incident on just one 3-edge of $P$.

Proof. Let $e_{1}, e_{2}, e_{3}$ and $e_{4}$ be the four edges of Boundary $(q)$ that are incident on some vertex $v$. We will prove the lemma by showing that the number of $e^{\text {s's }}$ which are 3-edges of $P$ is even. For $1 \leq i \leq 4$, let $f\left(e_{i}\right)$ be the number of 2 -xels in $P$ that have $e_{i}$ as an edge (so that $f\left(e_{i}\right)=0,1,2$ or 3 ). Now $f\left(e_{i}\right) \neq 1$ (otherwise $e_{i}$ would be a free face of $P$ ), so $f\left(e_{i}\right)$ is odd if and only if $e_{i}$ is a 3 -edge of $P$.

Note that $f\left(e_{1}\right)+f\left(e_{2}\right)+f\left(e_{3}\right)+f\left(e_{4}\right)$ is even since each 2 -xel incident on $v$ is incident on exactly two of the e's and is therefore counted just twice in this sum, and 2-xels not incident on $v$ are not incident on any $e$ and are not counted. Hence the number of $f\left(e_{i}\right)$ 's that are odd is even, and so the number of $e_{i}$ 's that are 3-edges of $P$ is even.

Corollary 17. In each of (a) and (b) below, the thick black edges cannot all be 3-edges of $\operatorname{Schlegel}(P)$ :

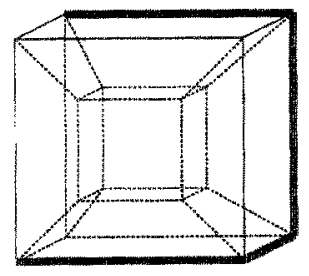

(a)

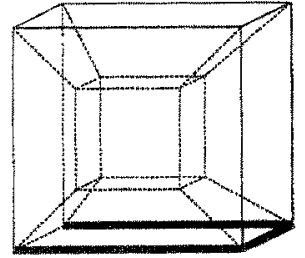

(b)

Proof. The thick black edges in (a) cannot all be 3-edges of Schlegel $(P)$ by Lemma 14. If the thick black edges in (b) were all 3-edges of Schlegel $(P)$ then by Lemma 16 all four thick black edges in one of the cases of Corollary 15 would be 3-edges of Schlegel $(P)$, contrary to that result.

Now we suppose $P$ has a 3 -edge and deduce a contradiction. By Lemma 16 , Corollary 17 and symmetry, we may assume the three light gray edges in the left-hand diagram of Figure 9 are 3 -edges of $\operatorname{Schlegel}(P)$. Then it follows, again from Lemma 16 and Corollary 17 , that the five thick black edges in that diagram are also 3-edges of Schlegel $(P)$. Hence the 2-cell $a$ in the right-hand diagram of Figure 9 is in Schlegel $(P)$ (since $a$ is incident on a 3-edge of Schlegel $(P)$ ). 

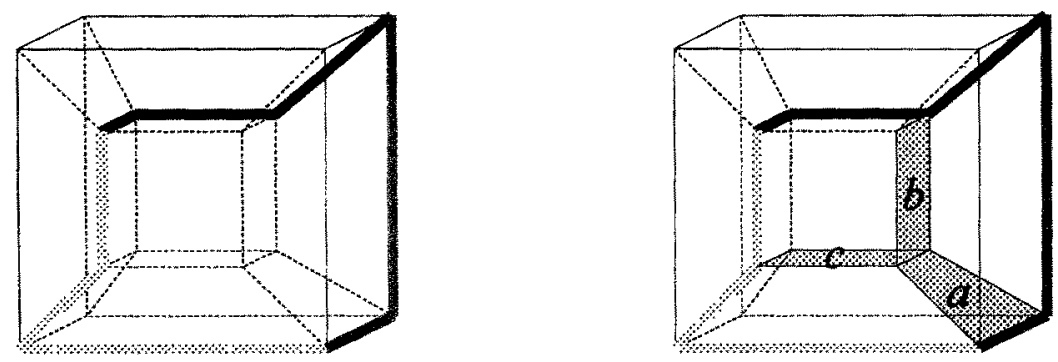

Fig. 9. If $P$ has a 3-edge then we may assume the three gray edges in these diagrams are 3-edges of Schlegel $(P)$. This implies that the five thick black edges are also 3-edges of Schlegel $(P)$, and hence that $a$ and either $b$ or $c$ in the right-hand diagram are in Schlegel $(P)$. But this leads to a contradiction of Lemma 14.

As $a$ is in Schlegel $(P)$ and $P$ has no free faces, at least one of the 2-cells $b$ and $c$ is also in Schlegel $(P)$. Four of the six 2-cells that are faces of the inner 3-cell in these diagrams are incident on a 3-edge of $\operatorname{Schlegel}(P)$, and are therefore in Schlegel $(P)$. Since either $b$ or $c$ must also be in Schlegel $(P)$, we see that at least five of the six 2-cells that are faces of the inner 3-cell are in Schlegel $(P)$. This contradiction of Lemma 14 proves that there are no 3-edges of $P$ and completes the proof of the Main Theorem in the case $n=4$.

\section{Another Discrete Characterization of Simple Xels.}

A. W. Roscoe and the author have discovered another discrete characterization of simple 1's in $n$-dimensional binary images, for $n \leq 4$. To state this characterization, define a generalized image to be a set $I$ of xels such that if $x$ is any xel in $I$ then no face of $x$ is in $I$. (Note that an $n$-dimensional binary image is a generalized image, for any positive integer $n$. A xel complex is not a generalized image unless it consists only of 0-xels, but the set of maximal xels of any xel complex is a generalized image.)

If $q$ is any xel in a generalized image $I$, then the attachment image of $q$ in $I$, denoted by ati $(q, I)$, is defined to be the generalized image given by the set of all the maximal xels in Boundary $(q) \cap \bigcup\{\operatorname{Boundary}(x) \mid x \in I-\{q\}\}$.

Definition 18. A xel $q$ in a generalized image $I$ is inductively simple in $I$ if ati $(q, I) \neq \emptyset$ and the xels in ati $(q, I)$ can be arranged in a sequence $a_{0}, a_{1}, \ldots, a_{k}$ such that, for $0 \leq i \leq k-1, a_{i}$ is inductively simple in the generalized image ati $(q, I)-\left\{a_{j} \mid 0 \leq j<i\right\}$. (Note that this defining condition is vacuously true if ati $(q, I)$ consists of just one xel; this is the base case of the inductive definition.)

Theorem 19 (Kong and Roscoe). For $n \leq 4$, a 1 in an n-dimensional binary image $I$ is simple in $I$ if and only if it is inductively simple in $I$. 
A proof of this theorem will be given elsewhere [5]. Although the theorem is stated only for $n \leq 4$, the result may well hold for some larger values of $n$. But we know it fails for $n \geq 24$ [5].

\section{Conclusion.}

For $n \leq 4$, the Main Theorem (Theorem 7) gives necessary and sufficient conditions for a 1 in an $n$-dimensional binary image $I$ to be simple; if we know which of the $3^{n}-1$ neighbors of a given 1 of $I$ are also 1 's of $I$, then it is easy to use these conditions to determine, by visual inspection, whether the given 1 is simple. An alternate version of the Main Theorem (Theorem 9) gives a practical algorithm for solving the 4-dimensional case of this problem.

A different discrete characterization of simple 1's in binary images of dimension $\leq 4$, due to the author and $\mathrm{A}$. W. Roscoe, has also been stated.

\section{Acknowledgment.}

The 4-dimensional case of the Main Theorem was conjectured to the author by A. W. Roscoe in 1995. The author is pleased to acknowledge stimulating discussions with Prof. Roscoe, in person and by e-mail, on this topic.

\section{References}

1. M. A. Armstrong, Basic Topology, Springer-Verlag, New York, 1983.

2. S. S. Cairns, Introductory Topology, Ronald Press, New York, 1968.

3. T. Y. Kong, On topology preservation in 2-D and 3-D thinning, International Journal of Pattern Recognition and Artificial Inteligence 9, 1995, 813-844.

4. T. Y. Kong and A. W. Roscoe, Characterizations of simply-connected finite polyhedra in 3-space, Bulletin of the London Mathematical Society 17, 1985, 575 - 578.

5. T. Y. Kong and A. W. Roscoe, Simple Points in 4-Dimensional (and HigherDimensional) Binary Images. Paper in preparation.

6. G. Malandain and G. Bertrand, Fast characterization of 3D simple points, Proceedings, 11th IAPR International Conference on Pattern Recognition, Volume III, The Hague, The Netherlands, 1992, $232-235$.

7. A. Rosenfeld, Connectivity in digital pictures, J. ACM 17, 1970, 146-160.

8. P. K. Saha, B. Chanda and D. D. Majumder, Principles and Algorithms for $2 D$ and $3 D$ Shrinking, Technical Report TR/KBCS $/ 2 / 91$, N. C. K. B. C. S. Library, Indian Statistical Institute, Calcutta, India, 1991.

9. Y.F. Tsao and K. S. Fu, A 3D parallel skeletonwise thinning algorithrn, Proceedings, IEEE Computer Society Conference on Pattern Recognition and Image Processing, $1982,678-683$. 